\title{
On the Canonical Transformation of Time-Dependent Harmonic Oscillator
}

\author{
Akpan N. Ikot, ${ }^{1}$ Louis E. Akpabio, ${ }^{1}$ Ita O. Akpan, ${ }^{2}$ \\ Michael I. Umo, ${ }^{2}$ Oladunjoye A. Awoga, ${ }^{1}$ and Alice B. Nyong ${ }^{1}$ \\ ${ }^{1}$ Department of Physics, University of Uyo, P.M.B 1017, Uyo, Nigeria \\ ${ }^{2}$ Department of Physics, University of Calabar, P.M.B 1115, Calabar, Nigeria \\ Correspondence should be addressed to Akpan N. Ikot, ndemikot2005@yahoo.com
}

Received 24 June 2009; Accepted 5 November 2009

Academic Editor: Nicholas Bigelow

Copyright () 2010 Akpan N. Ikot et al. This is an open access article distributed under the Creative Commons Attribution License, which permits unrestricted use, distribution, and reproduction in any medium, provided the original work is properly cited.

We performed a two-variable canonical transformation on the time momentum operator, and without loss of generality we carried out a three-variable transformation on the coordinate and momentum space operators to trivialize the Hamiltonian operator of the system. Fortunately, this operation separates the time-coordinate and space coordinate naturally, and the wave function of the time-dependent Harmonic Oscillator is evaluated via the generator.

\section{Introduction}

The method of Canonical transformations (CT) has proved to be a fruitful approach in treating quantum systems [1]. It is often used in describing systems with Hamiltonian that are quadratic either in co-ordinate and momentum or equivalently in Boson creation and annihilation operators. One major advantage of the method of (CT) consists in reducing the Hamiltonian $H$ of the system $S$ to a Hamiltonian $H^{\prime}$ of some simple system $S^{\prime}$ with known solution, that is, $H=$ $\mathrm{CH}^{\prime} \mathrm{C}^{-1}$. These two systems are canonically equivalent since their Hamiltonian is related by means of a CT. However, analytical approaches to isolate conserved quantities for a given physical system are a major objective in the realm of Hamiltonian theory [2]. For autonomous, where the Hamiltonian is time independent, one conserved quantity is immediately found: the Hamiltonian which represents the total energy of the system is a constant of motion.

Rather unfortunately the Hamiltonian of most real physical systems is explicitly time-dependent and does not provide direct conserved quantity. In classical mechanics, the equation of motion governed the dynamical behavior of the system and this second-order differential equation is solved directly by the trivialization of the conjugate variables through CT [3]. Park [3] asserted that because of the noncommutability of operators, CT has not been fully realized in quantum mechanics. However, CT is usually defined as a change of the noncommutating variables that preserved the commutation relations in quantum mechanics [4].

In literature $[4,5]$ three types of transformations have been identified such as interchange, similarity, and point transformations. Different models of oscillators have been evaluated via the CT $[6,7]$. The applications of quantum canonical transformation to a harmonic oscillator in which the angular frequency and equilibrium position are time dependent have been reviewed [3]. In this paper we extend the work of [3] by performing a three-variable transformation on both the space co-ordinate and momentum coordinate operators.

\section{Time-Dependent Harmonic Oscillator}

The Time-Dependent Schrodinger Wave equations have been solved explicitly by the method of Quantum Canonical Transformation (QCT). The QCT is also used in solving time-dependent frequency [3]. However, the solutions of time-dependent harmonic oscillator have been obtained through various methods including invariant operator [8], Path Integral $[9,10]$, and the space-time transformation $[11,12]$. We write the Schrödinger equation to be solved as

$$
\hat{H}_{1} \Psi_{1}(q, t)=0,
$$


where

$$
H_{1}=P_{t}+P^{2}+\Omega^{2}(t)\left(q-x_{0}(t)\right) \text {, }
$$

with $P_{t}=-i \partial / \partial t$, and $P=-i \partial / \partial q$, as the momentum operators in time and in the co-ordinate. It is worthy to note that the angular frequency $\Omega(t)$ and the equilibrium position $x_{0}(t)$ are all functions of time.

We make time-variable similarity transformation on the momentum time-coordinate and three-variable transformations on space momentum operators as

$$
P_{t} \longrightarrow P_{t}-\frac{i}{2}\left(\frac{\partial f}{\partial t}\right) q^{2}, \quad P \longrightarrow P-i f q-\frac{i}{2}\left(\frac{\partial f}{\partial q}\right) .
$$

And the Hamiltonian Operator becomes

$$
\begin{aligned}
H_{2}= & P_{t}-\frac{i}{2}\left(\frac{\partial f}{\partial t}\right) q^{2}+\left(P-i f q-\frac{i}{2}\left(\frac{\partial f}{\partial q}\right)\right)^{2} \\
& +\Omega^{2}(t)\left(q-x_{0}(t)\right)^{2} \\
= & P_{t}+P^{2}+\left(\Omega^{2}(t)-\frac{i}{2}\left(\frac{\partial f}{\partial t}\right)-f^{2}\right) q^{2} \\
& -\left(i P \frac{\partial f}{\partial q}+f q \frac{\partial f}{\partial q}+\frac{1}{4}\left(\frac{\partial f}{\partial q}\right)^{2}\right) \\
& -2 \Omega^{2}(t) x_{0}(t) q+\Omega^{2}(t) x_{0}(t)-f .
\end{aligned}
$$

The condition for the differential in (4) to be cancelled is

$$
4 \frac{\partial^{2} f}{\partial q^{2}}+4 f q\left(\frac{\partial f}{\partial q}\right)+\left(\frac{\partial f}{\partial q}\right)^{2}=0
$$

And we solve (5) by series method as

$$
f(q)=\sum_{k} C_{k} q^{k+\beta}
$$

where $C_{k}$ is the expansion coefficient and $K$ and $\beta$ are arbitrary constant.

Substituting (6) into (5) yields

$$
f(q)=q^{2}+2 i \sqrt{2} q+8 .
$$

Imposing boundary condition on (7), we obtain $f(0)=8$ and (7) becomes

$$
f(q)=q^{2}+2 i \sqrt{2} q+f(0)
$$

And we recaste (8) in the form

$$
f(q)=q^{2}+2 i \sqrt{2} q,
$$

where $f(0)$ has been absorbed into $f(q)$. Now multiplying the first term of the LHS of (9) by $(-1 / 2) m \omega^{2}$ and replacing the magnitude $2 i \sqrt{2}$ by $f(q)$ and replacing $f \rightarrow L-$ $(1 / 2) m \dot{q}^{2},(9)$ becomes

$$
L=\frac{1}{2} m \dot{q}^{2}-\frac{1}{2} m \omega^{2} q^{2}+F(q) q
$$

and (10) is the Lagrangian of the system whose classical equation is

$$
m \ddot{q}+m \omega^{2} q-\frac{\partial V}{\partial q}=0
$$

The result of (10) shows that the cancellation of the differential in (4) generates the Lagrangian describing the harmonic oscillator of the system. Another condition of (4) is the cancellation of quadratic term in co-ordinate and these yields

$$
i \frac{\partial f}{\partial t}+2 f^{2}=2 \Omega^{2}(t)
$$

however (12) is a first-order nonlinear equation and can be linearized by setting

$$
f=\frac{i}{2} \frac{\partial}{\partial t} \ln \phi(t)
$$

and (12) becomes

$$
\frac{\partial^{2}}{\partial t^{2}} \phi(t)+4 \Omega^{2}(t) \phi(t)=0
$$

Solving (14) gives

$$
\phi(t)=\phi(0) \sin (\omega t+\delta)
$$

where $\delta$ is the phase factor. Putting (15) into (13) leads to

$$
f(t)=\frac{i \omega}{2} \tan (\omega t+\delta) .
$$

With (16) and (9), we can determine the generator $e^{-f(q)} e^{-f(t)\left(q^{2} / 2\right)}$.

The second Canonical transformation is carried out this time to remove the quadratic term in momentum and the associated differential in co-ordinate:

$$
P_{t} \quad \longrightarrow P_{t}-\frac{i}{2}\left(\frac{\partial g}{\partial t}\right) P^{2}, \quad q \longrightarrow q-i g P+\frac{i}{2}\left(\frac{\partial g}{\partial q}\right) .
$$

Substituting (17) into (4) yields

$$
\begin{aligned}
H_{3}= & P_{t}+\left(2+4 g f-i \frac{\partial g}{\partial t}\right) \frac{P^{2}}{2}-\left(i f \frac{\partial^{2} g}{\partial q^{2}}+i \Omega^{2}(t) \frac{\partial g}{\partial q}\right) \\
& -2 i f g p-2 i \Omega^{2} x_{0}(t) g P-2 \Omega^{2} x_{0}(t) P+\Omega^{2} x_{0}^{2}-f
\end{aligned}
$$

We eliminate the quadratic term in momentum by setting

$$
2+4 g f-i \frac{\partial g}{\partial t}=0
$$

and solving (19), we obtain

$$
g(t)=-2 i e^{-4 i \int f(t) d t} \int e^{4 i \int f(t) d t}+K e^{-4 i \int f(t) d t} d t .
$$


Similarly, the differential in co-ordinate is eliminated as

$$
\frac{\partial^{2} g}{\partial q^{2}}+\frac{\Omega^{2}(t)}{f} \frac{\partial g}{\partial q}=0
$$

with $f$ being known, and we determined (21) as

$$
g(t)=D+F e^{-\alpha^{2}(q) \omega^{2} q},
$$

where $D$ and $F$ are arbitrary constants and $\alpha(q)=1 / f(q)$.

The next transformation is carried out to eliminate the linear term in position $q$ and the associated differential in co-ordinate:

$$
P_{t} \longrightarrow P_{t}-i\left(\frac{\partial h}{\partial t}\right) q, \quad q \rightarrow q-i h+\frac{i}{2}\left(\frac{\partial h}{\partial q}\right),
$$

and the Hamiltonian becomes

$$
\begin{aligned}
& H_{4} \\
& =P_{t}-\left(i \frac{\partial h}{\partial t}+2 f h+2 \Omega^{2} x_{0}(t)\right) q-\left(f q \frac{\partial h}{\partial q}+\Omega^{2} x_{0} g \frac{\partial h}{\partial q}\right) \\
& \quad-2 i f q p-2 i \Omega^{2} x_{0} g p-2 \Omega^{2} x_{0} g h+\Omega^{2} \mathrm{x}_{0}^{2}-f .
\end{aligned}
$$

The condition for the linear term in $q$ and the differential to be cancelled leads to the solution of $h(t)$ as

$$
h(t)=2 i e^{2 i \int f(t) d t} \int \Omega^{2} x_{0} e^{-2 i \int f(t) d t} d t,
$$

and the $h(q)$ as

$$
h(q)=C e^{\left(\int f(g)[\ln (q)]^{-1} / g \Omega^{2}\right) d q},
$$

where $C$ is an arbitrary constant.

Another two- and three-variable transformation is carried out on the Hamiltonian to eliminate the linear term in momentum and the associated differential in co-ordinate:

$$
P_{t} \longrightarrow P_{t}-\frac{i}{2}\left(\frac{\partial r}{\partial t}\right) P, \quad q \longrightarrow q-i r+\frac{i}{2}\left(\frac{\partial r}{\partial q}\right)
$$

and the Hamiltonian becomes

$$
\begin{aligned}
H_{5}= & P_{t}-\left(\frac{i}{2} \frac{\partial r}{\partial t}+2 i \Omega^{2} x_{0} g-2 f r\right) P-2 i f q p \\
& +i f \frac{\partial^{2} r}{\partial q^{2}}-2 \Omega^{2} x_{0} g h+\Omega^{2} \mathrm{x}_{0}^{2}-f .
\end{aligned}
$$

The linear term in $P$ in (28) is eliminated if

$$
\frac{i}{2}\left(\frac{\partial r}{\partial t}\right)+2 i \Omega^{2} x_{0} g-2 f r=0
$$

and solving (29) yields

$$
r(t)=2 e^{2 i \int f(t) d t} \int \Omega^{2} x_{0} g e^{-2 i \int f(t) d t} d t+C e^{-2 i \int f(t) d t},
$$

and the condition for the differential in co-ordinate to cancelled yields

$$
\frac{\partial^{2} r(q)}{\partial q^{2}}=0
$$

However (31) is a second-order differential Equation and we rewrite (31) in the form

$$
\frac{i f}{q^{2}} \frac{d}{d q}\left\{q^{2} \frac{\partial r}{\partial q}\right\}=0
$$

or

$$
\frac{\partial^{2} r}{\partial q^{2}}+\frac{1}{q} \frac{\partial r}{\partial q}=0
$$

Multiplying (33) by $q^{2}$ gives

$$
q^{2} \frac{\partial^{2} r}{\partial q^{2}}+q \frac{\partial^{2} r}{\partial q}=0
$$

and comparing (34) with (35) we have

$$
q^{2} y^{\prime \prime}+x y^{\prime}-\lambda^{2} y=0
$$

This shows that (34) is a Bessel equation with $\lambda=0$. The general solution of (33) becomes

$$
r(q)=A_{1} J_{o}(m q)+A_{2} Y_{o}(m q)
$$

where $m$ is an integer, $A_{1}, A_{2}$ are arbitrary constant, and $J_{o}(q)$ and $Y_{o}(q)$, respectively, represent Bessel functions of first and second kind.

Again a point transformation is done to eliminate the coordinate in the Hamiltonian of (28) [3]:

$$
P \longrightarrow e^{-q} P, \quad q \longrightarrow e^{q} .
$$

Substituting (37) into (28) will be

$$
H_{6}=P_{t}-2 i f p-2 \Omega^{2} x_{0} g h+\Omega^{2} \mathrm{x}_{0}^{2}-f .
$$

In order to trivialize the Hamiltonian of (38), we carried out a two-variable similarity transformation:

$$
P_{t} \longrightarrow P_{t}+2 i f p+2 \Omega^{2} x_{0} g h-\Omega^{2} \mathrm{x}_{0}^{2}+f,
$$

and (38) becomes

$$
H_{7}=P_{t}
$$

The Schrodinger equation associated with the trivialized Hamiltonian of (40) is

$$
P_{t} \Psi_{7}^{(q)}=H_{7} \Psi_{7}^{(q)}
$$

where $\Psi_{7}^{(q)}$ being the time-dependent Wave function originates from the differential in the canonical transformed Hamiltonian Operators. 
The complete wave function $\Psi_{1}(q, t)$ is obtained as

$$
\begin{aligned}
\Psi_{1}(q, t)= & \Psi(q) \Psi(t) \\
= & \mathrm{e}^{f(q)} \mathrm{e}^{g(p)} \mathrm{e}^{h(p)} \mathrm{e}^{\gamma(p)} \mathrm{e}^{f(t) q^{2} / 2} \mathrm{e}^{g(t) p^{2} / 2} \mathrm{e}^{h(t) P} \mathrm{e}^{\gamma(t) \mathrm{P}} \\
& \times \mathrm{P}_{\ln g} \mathrm{e}^{-2 P \int f(t) d t+i \int\left(2 \Omega^{2} x_{0} g h-\Omega^{2} \mathrm{x}_{0}{ }^{2}+f\right) d t} .
\end{aligned}
$$

The first four terms of (42) are the times Independent Wave Function arising naturally from the cancellation of the differential in co-ordinate in the Hamiltonian Operator while the other terms are the time-dependent wave function gotten as the consequences of the cancellation in the co-ordinates and momentum.

Solving (42) completely yields the result as [3] without the ambiguity of $\Psi_{1}(q)$ which they encountered because $\Psi_{1}(q)$ is obtained directly from the cancellation of the differentials in the Hamiltonian.

We obtained after a simple algebra the unnormalized wave function from (11) as

$$
\begin{aligned}
& \Psi_{1}(q, t) \\
& =N g^{3 n / 2} \mathrm{e}^{-(1 / 2) g h^{2}} \exp \left(i \int\left[2 \omega^{2} x_{0} g h-\omega^{2} x_{0}^{2}+(2 n+1) f\right] d t\right) \\
& \quad \times e^{(1 / 2) f q^{2}+h q} H_{n}\left(\sqrt{\frac{g}{2}}\left(\frac{q-i \gamma}{g}-h\right)\right),
\end{aligned}
$$

where $N$ is the normalization constant and $H_{n}(\sqrt{g(q) / 2}(q-$ $i \gamma(q) / \Omega-h(q)))$ is the Hermite polynomial of order $n$.

\section{Conclusion}

The introduction of a three-variable CT on the co-ordinate and the momentum space removed the ambiguity encountered in [3] in evaluating the time-independent Schrödinger wave function. In (41), we obtain the generalized wave function of the time dependent harmonic oscillator with the first four terms arising from the introduction of the three canonical variables. These terms were simply given as $\Psi_{1}$ in [3] without its explicit values as we have obtained above. Rather fortunately the three-variable transformations separate naturally the time-dependent and time-independent function explicitly. This separation aids us to solve the Schrödinger equation completely by trivializing the timedependent Hamiltonian Operator. In addition our result can be viewed as a generalized and explicit case of that obtained in [3].

The extension of this work to different time dependent systems with different potential is possible. Thus canonical transformation is a good tool in solving time-dependent systems.

\section{Acknowledgment}

The authors acknowledge the Nandy-Leabio Foundation for partial financial support in this work.

\section{References}

[1] D. A. Trifonor, "Diagonalization of hamiltonians, uncertainty matrices and Robertson inequality," in Proceedings of the 2nd International Conference on Geometry, Integrability and Quantization, Varna, Bulgaria, June 2000.

[2] J. Struckmeier and C. Riedel, "Direct validation technique for numerical simulations of time-dependent Hamiltonian systems," Physical Review E, vol. 64, Article ID 0265503, 2001.

[3] T. J. Park, "Canonical transformation for time dependent harmonic oscillator," Bulletin of the Korean Chemical Society, vol. 25, p. 2, 2004.

[4] A. Anderson, Journal of Physics A, vol. 232, p. 293, 1994.

[5] J. Deenen, "Generators for nonlinear canonical transformations," Journal of Physics A, vol. 24, pp. 3851-3858, 1991.

[6] G. Y. Tsaur and J. Wang, "Integration of the Schrödinger equation by canonical transformations," Physical Review A, vol. 65, Article ID 012104, 7 pages, 2001.

[7] T. J. Park, "Exactly solvable time dependent problems: potential of monotonously decreasing function of time," Bulletin of the Korean Chemical Society, vol. 23, p. 1733, 2002.

[8] H. R. Lewis Jr., "Class of exact invariants for classical and quantum time-dependent harmonic oscillators," Journal of Mathematical Physics, vol. 9, no. 11, pp. 1976-1986, 1968.

[9] A. N. Ikot, E. E. Ituen, I. E. Essien, and L. E. Akpabio, "Path integral evaluation of a time-dependent oscillator in an external field," Turkish Journal of Physics, vol. 32, no. 6, pp. 305-313, 2008.

[10] C. I. Um, K. H. Yeon, and T. F. George, "The quantum damped harmonic oscillator," Physics Reports, vol. 362, no. 2-3, pp. 63192, 2002.

[11] M. Feng, "Complete solution of the Schrödinger equation for the time-dependent linear potential," Physical Review A, vol. 64, Article ID 034101, 3 pages, 2001.

[12] T. J. Park, "Wave function of time dependent harmonic oscillators," Bulletin of the Korean Chemical Society, vol. 23, p. 355,2000 . 

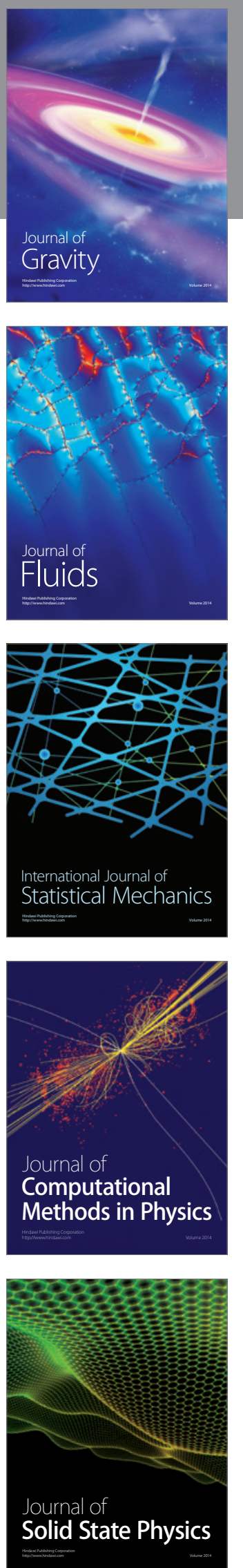

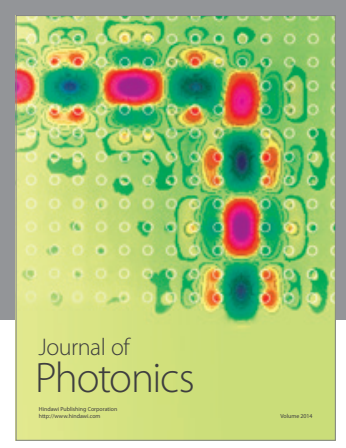

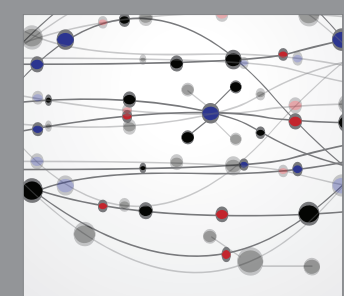

The Scientific World Journal
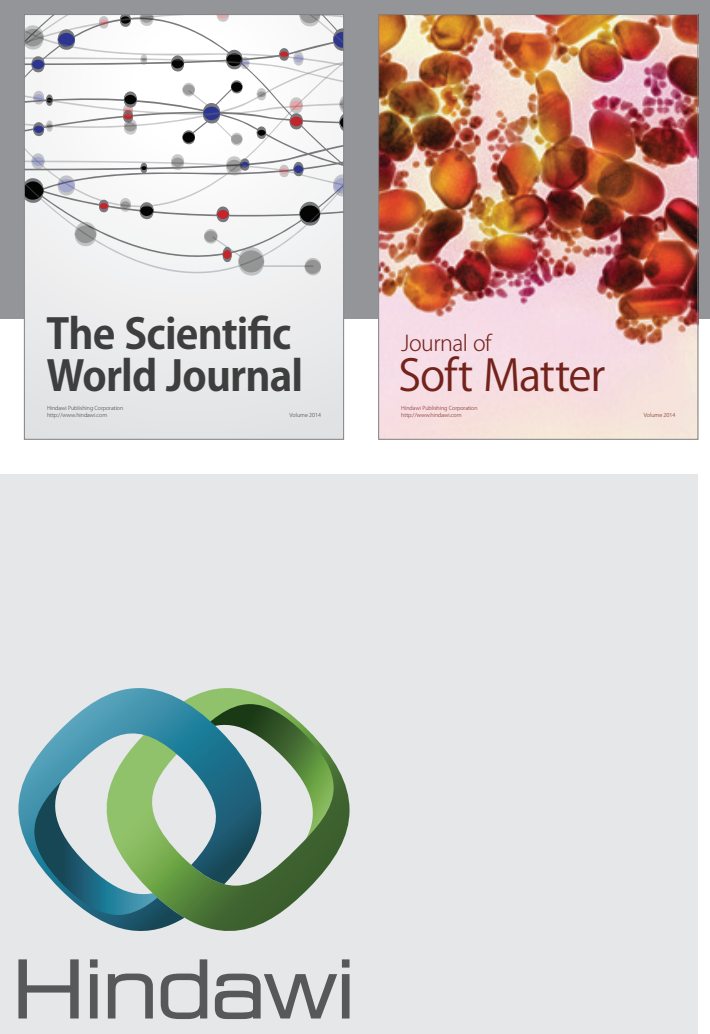

Submit your manuscripts at

http://www.hindawi.com
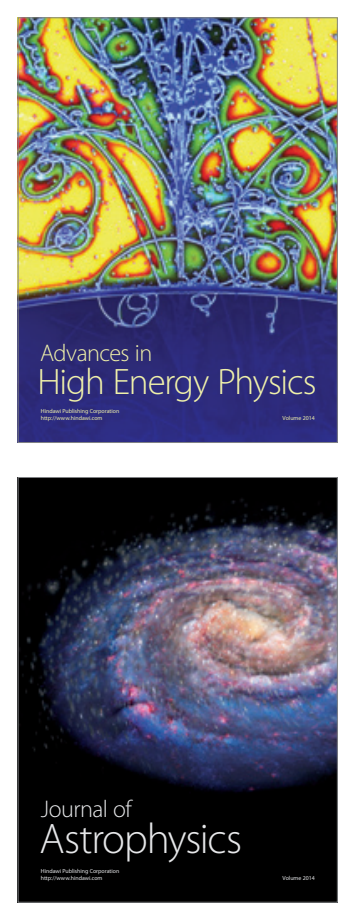
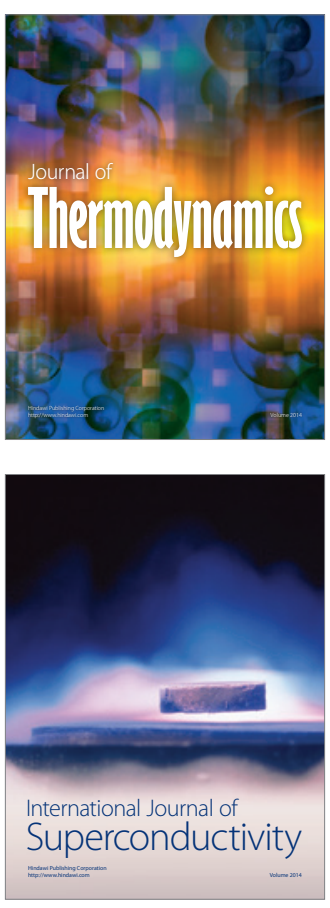
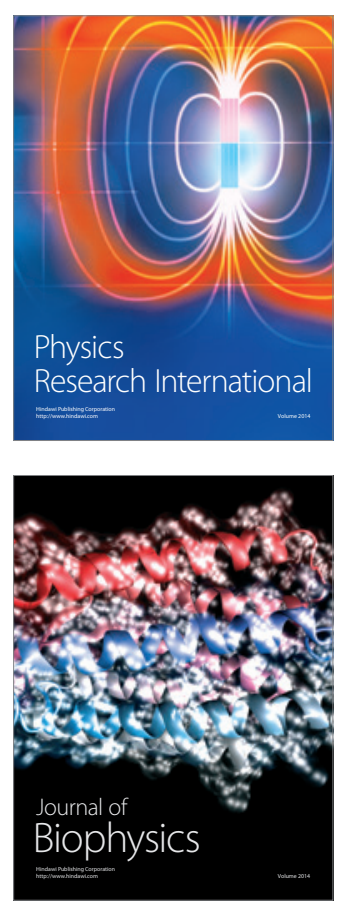
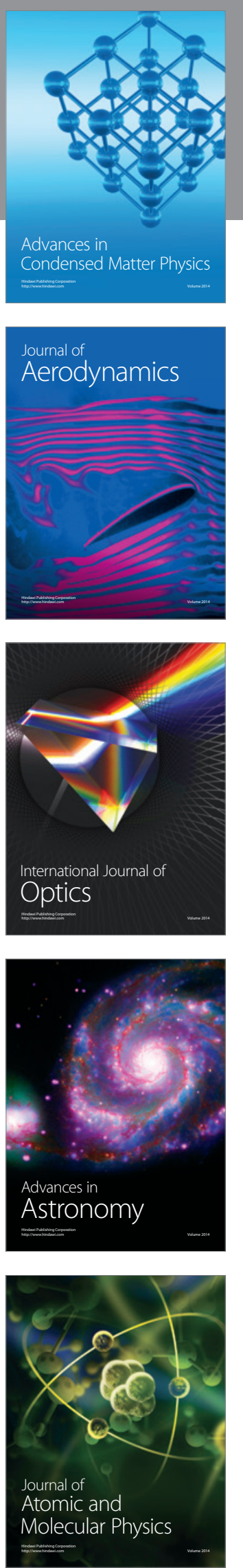